\title{
Modeling of the polymerization process of the insulating layer of cable thermocouples
}

\author{
Iashutina Olga ${ }^{1, *}$, Asadullina Liliya ${ }^{1}$, and Chernova Galina $^{1}$ \\ ${ }^{1}$ National Research Tomsk Polytechnic University, 634050 Tomsk, Russia
}

\begin{abstract}
A mathematical model describing the main physical processes occurring during thermal processing of cable thermocouples (CT) during their production is presented. The polymerization of the insulating layer is taken into account in the conditions of its intense heating. The developed mathematical model allows to carry out calculations of heat transfer processes under the conditions of substantial heterogeneity of cable thermocouple structure. The computational experiments carried out have established significant differences in the characteristics of heat transfer in $\mathrm{CT}$ in the presence of thermoelectrodes with differing thermophysical characteristics.
\end{abstract}

\section{Introduction}

One of the most important controlled parameters of technological processes in chemical [1], petrochemical [2], energy [3], fuel, and other industries is temperature. The latter is the most informative characteristic that determines the entire physics of the technological process.

At present, two main methods for measuring temperature have been developed: contact (with the help of thermocouples, resistance thermometers, liquid thermometers, etc.) [4] and non-contact (using a pyrometer) [5]. The most preferred method of temperature measurement is the contact method. The latter is due to the cheapness and simplicity of the corresponding equipment of contact methods in comparison with non-contact methods. The most common of contact methods for measuring temperature is thermocouple [6]. The thermocouple is a sensor that consists of two interconnected dissimilar electroconductive elements, usually metallic conductors or sometimes semiconductors [7].

Unlike conventional industrial thermocouples in a sturdy ceramic housing, the cable thermocouple has such advantages as the flexibility and tightness of the housing [8]. Cable thermocouples are used in a very wide temperature range (from $23 \mathrm{~K}$ to $2800 \mathrm{~K}$ ) [9] in all industries (from cryogenic technology to high-temperature heat engineering installations).

At present, in spite of the large-scale experimental [10] and theoretical [11] studies of heat and mass transfer processes that take place in the manufacture of thermocouple products, the physical theory and the mathematical basis for the thermal processes that occur in the manufacture of cable thermocouples have not been developed yet. The latter is

Corresponding author: osy1@tpu.ru 
due to the complexity of calculating the characteristics of heat transfer processes occurring under conditions of intense thermochemical (polymerization) transformations in a small (up to $1 \cdot 10^{-3} \mathrm{~m}$ ) one.

The purpose of the work: mathematical modeling of heat transfer processes proceeding together under conditions of intense radiation-convective heat transfer in the manufacture of cable thermocouples.

\section{Problem statement}

In the formulation of the problem, it was assumed that at the initial instant of time the cable thermocouple enters the high-temperature medium (intracameral space of the polymerization furnace). Under the influence of intense radiation-convective heat transfer, its heating occurs. As a result, the process of polymerization of the internal insulation of the cable thermocouple is initiated. It should be noted that thermoelectrodes with different thermophysical characteristics (thermal conductivity $\lambda$, heat capacity $C$, density $\rho$ ) are symmetrical in relation to the thermocouple axis. Accordingly, it can be reasonably assumed that the location of the thermal center of symmetry will differ from the localization of the geometric center of the thermocouple. In mathematical modeling, the system in Figure 1 was considered.

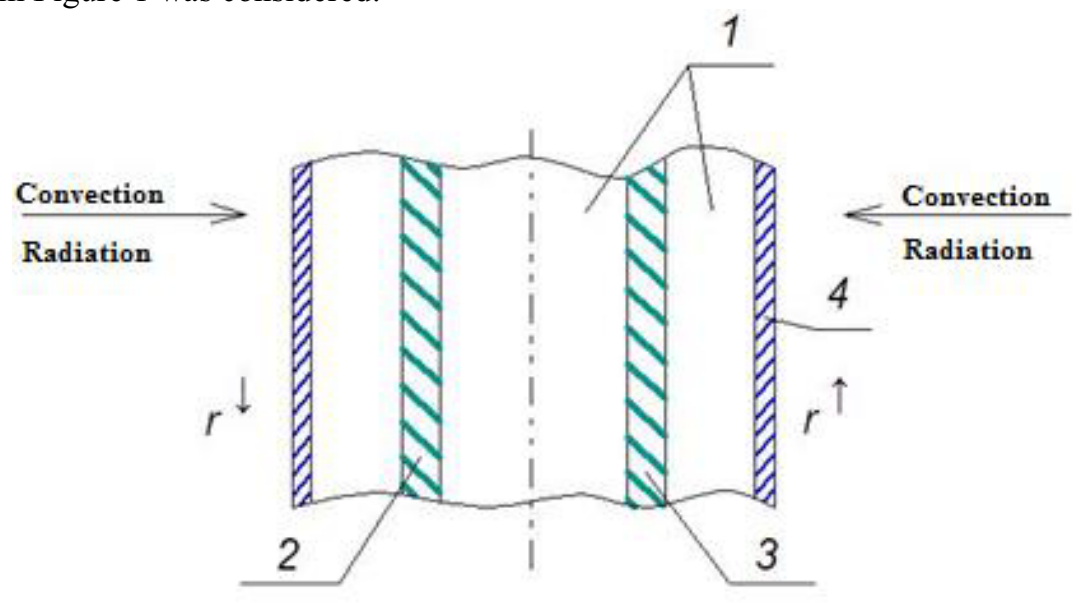

Fig. 1. Scheme of the problem solution area: 1 - insulation powder $(\mathrm{MgO}) ; 2,3$ - thermoelectrodes; 4 - cable thermocouple sheath.

\section{Results and discussion}

In order to verify the mathematical model, a test calculation of heat transfer was carried out with the thermophysical characteristics of thermoelectrodes being equal. The results of the computational experiments are shown in Fig. 2. It can be noted that the temperature curve is a parabola with a center of symmetry on the axis of the thermocouple. The latter testifies to the physical nature of the calculation results. Accordingly, we can say that the developed mathematical model conducts a prognostic analysis of heat transfer processes under conditions of intense heating of CT with a sufficiently high degree of accuracy. 


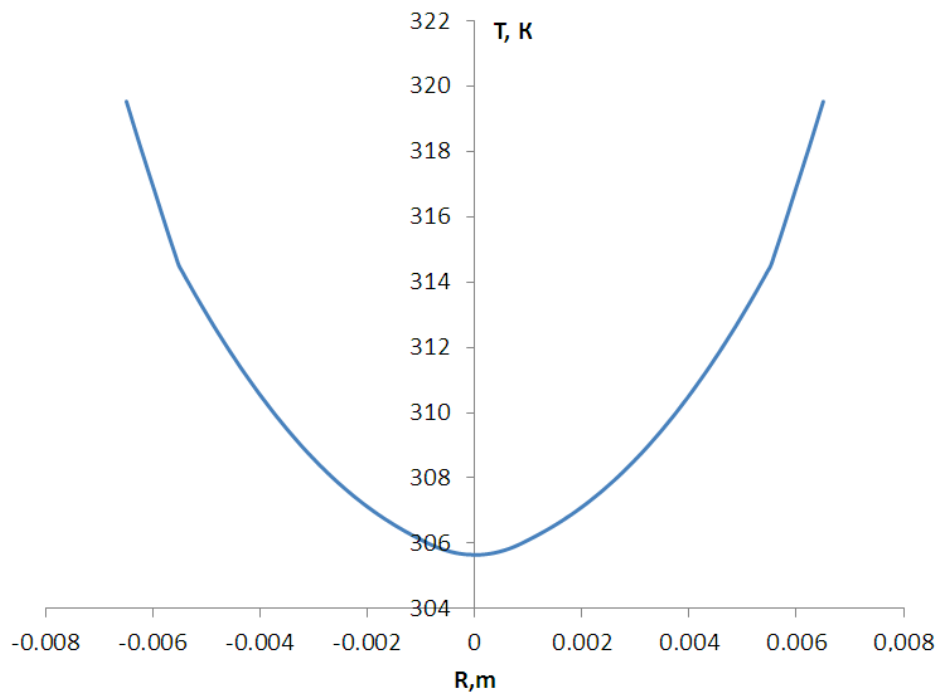

Fig. 2. The parabolic temperature curve.

Figure 3 shows the temperature dependence on the material of the cable thermocouple thermoelectrodes.

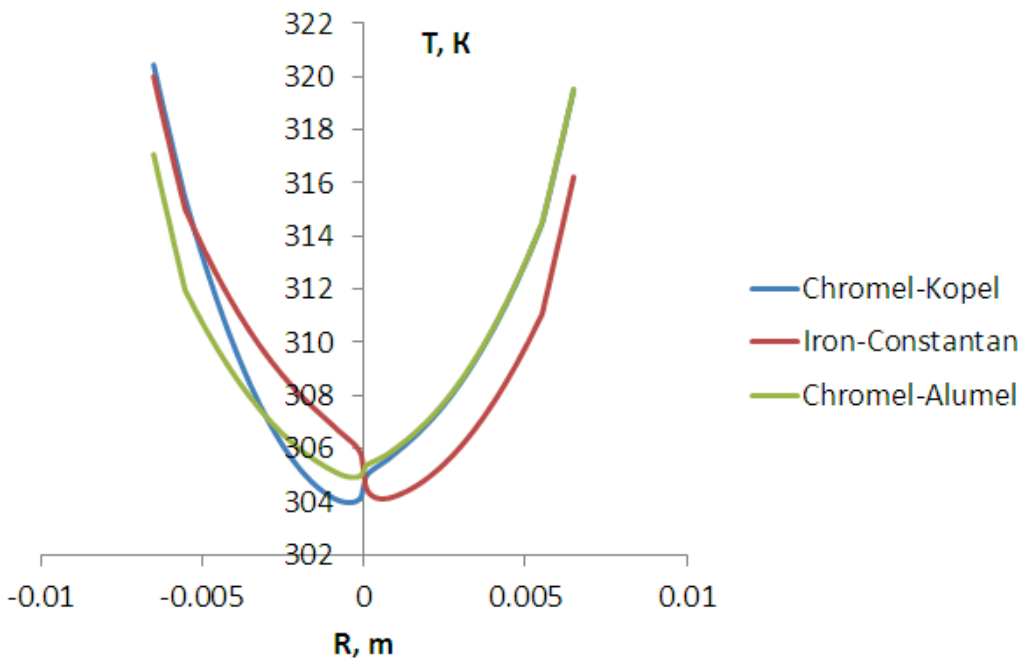

Fig. 3. Temperature distribution for different thermoelectrode materials.

Analyzing the obtained dependences, it can be noted that the temperatures at the boundaries of the "cable thermocouple-external environment" system $\left(r_{0}^{\downarrow}<r<r_{0}^{\uparrow}\right)$ are different. The latter indicates significant differences in the heat transfer conditions in both parts relating to the thermocouple axis.

Figure 4 shows the distribution of the polymerization parameters along the radius of the system under consideration.

The highest degree of polymerization is observed on the surface of a cable thermocouple. This is due to the high heat flows at the outer boundary and the high thermal conductivity $(\lambda=250 \mathrm{~W} / \mathrm{m} \cdot \mathrm{K})$ of the aluminum shell. 


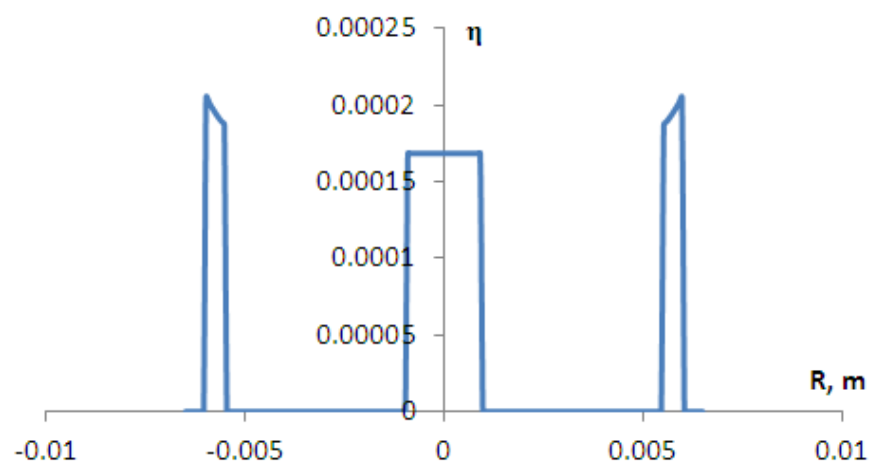

Fig. 4. Distribution of the polymerization parameters along the radius of the system.

\section{Conclusions}

For the first time, a mathematical model of the processes of heat and mass transfer proceeding together under conditions of intense thermochemical transformations (polymerization) when heating the cable thermocouples is presented. It should be noted that the displacement of the thermal center of symmetry relative to the axis of the thermocouple has been taken into account.

Based on the results of numerical modeling of heat transfer under conditions of radiation-convective heating, a significant inhomogeneity of the temperature field has been established, due to the difference in the thermophysical characteristics of thermoelectrodes. It was also shown that the degree of polymerization depends significantly on the location of the insulating layer relative to the center of the thermocouple.

\section{References}

1. M.V. Miroshkin, Exposition Oil Gas, 34 (2014)

2. I.S. Sheleba, Photon-Express, 6 (2015)

3. V.I. Nazarov, A.L. Burov, E.N. Kriskina, Thermotechnical measurements and devices (Tutorial, 2012) [in Russian]

4. E.V. Khaliulin, A.V. Kozlov, Bulletin of the Ufa State Aviation Technical University, 3 (2014) [in Russian]

5. A.V. Frunze, Adv. Powder. Metall. Part. Mater., 5, 40-42 (2009)

6. Yu.K. Atroshenko, I.P. Ozerova, P.A. Strizhak, Scientific and technical statements of SPbSPU. Electrical engineering, 214 (2015) [in Russian]

7. Yu.K. Atroshenko, P.A. Strizhak, Sensors and systems, 192 (2015)

8. V.A. Karzhavin, A.V. Karzhavin, Instrum Sci. Technol., 12, 12-22 (2010)

9. O.A. Geraschenko, A.N. Gordov, A.K. Eremina, Temperature measurements, (1989)

10. Y.K. Atroshenko, P.A. Strizhak, O.S. Yashutina, EPJ Web Conf., 82 (2015)

11. Y.K. Atroshenko, P.A. Strizhak, EPJ Web Conf., 76 (2014) 